\title{
Semiclassical Faddeev approximation for electron loss in fast heavy-ion collisions
}

\author{
D H Jakubassa-Amundsen \\ Physik-Department, Technische Universität München, Garching, Germany
}

Received 27 February 1981, in final form 16 April 1981

\begin{abstract}
The ionisation of the projectile in collisions with heavy targets is described by means of the Faddeev expansion. The series is cut off after five terms which means an inclusion of higher-order interactions of the electron with both the target and the projectile in a symmetric way. This approximation is compared with several theories suggested in the literature. Numerical calculations are presented for the energy distribution of electrons emitted in the forward direction in the case of $(\mathrm{H}, \mathrm{Ne})$ collisions.
\end{abstract}

\section{Introduction}

It is well known that total cross sections for ionisation in fast ion-atom collisions can be described within a first-order perturbation theory (McDowell and Coleman 1970). However, the Born approximation is not in general applicable for cross sections that are differential in the electronic degrees of freedom. When the momentum $\boldsymbol{k}_{f}$ of the ejected electron is such that the interaction with projectile (nuclear charge $\left.Z_{1}\right)$ and target $\left(Z_{2}\right)$ becomes equally important, it is no longer possible to include only the field of the parent nucleus to all orders. Examples are the double-differential cross section for the ejection of projectile electrons by heavy targets $\left(Z_{1} \ll Z_{2}\right)$ in the backward direction, where the observed structure in the angular distribution (Duncan and Menendez 1979) can only be explained when the electron is described as a target eigenstate (Jakubassa 1980). Also the 'forward peak', caused by target electrons that are ejected with a low velocity with respect to the projectile (Rudd et al 1966), can only be reproduced if an infinite number of interactions with the projectile are taken into account (Macek 1970). It has therefore been suggested that this process can be described as charge transfer to the continuum (Dettmann et al 1974). Several higher-order thories have been used for this purpose, such as the second-order Born approximation (Dettmann et al 1974, Miraglia and Ponce 1980), as well as a distorted wave Born approximation (Salin 1969, Garibotti and Miraglia 1980). One might also consider the impulse approximation (McDowell and Coleman 1970), which is well established for charge transfer into bound states (Briggs 1977, Jakubassa-Amundsen and Amundsen 1980) but which has not yet been applied to continuum charge transfer.

The phenomenon of this narrow forward peak also appears in electron-loss spectra (Duncan and Menendez 1979). Up to now there exists only a first-order theory to describe this behaviour of the projectile electrons (Drepper and Briggs 1976, Day 1980), and target properties have only been included by allowing for excitations of the target electrons (Briggs and Day 1980). 
The aim of this paper is to develop a theory for (projectile) ionisation which is valid over the whole range of electron momenta $\boldsymbol{k}_{f}$. Clearly, this theory must allow for interactions with both the projectile and the target to infinite order. Thereby it is convenient to start from the Faddeev equations for the exact electronic wavefunction (Faddeev 1961) and derive a systematic expansion in the projectile and target field. This approach was used by Macek (1970) who, however, retained only the first-order terms. The idea of including the interaction with the projectile and the target to higher order in a symmetric way has also been suggested by Briggs (1980) for the case of electron capture to bound states. For ionisation, however, one has the additional problem of not having a definite final electronic state, such that the Faddeev approach seems more appropriate than the $T$-matrix expansion he used. Section 2 contains the derivation of the Faddeev approximation, and in $\$ 3$ we discuss some approximations given in the literature. In $\S 4$ we calculate the energy distribution of electrons emitted in the forward direction in $(\mathrm{H}, \mathrm{Ne})$ collisions. We will show that even in this case a first-order theory is not sufficient for ionisation by heavy targets $(\S 5)$.

\section{The semiclassical Faddeev approximation (SCFA)}

Although electron loss is usually formulated within a full quantum mechanical theory we prefer the choice of the semiclassical approximation which is valid as long as a classical description of the internuclear motion can be justified. The advantage of this theory is the appearance of only electronic quantities in the matrix elements, although the translational factors arising from the transformation between the projectile and the target rest frame have to be included explicitly. The electronic part of the Hamiltonian is given by

$$
H=-\frac{\hbar^{2}}{2 m} \Delta+V_{\mathrm{P}}\left(\boldsymbol{r}_{\mathrm{P}}\right)+V_{\mathrm{T}}\left(\boldsymbol{r}_{\mathrm{T}}\right)
$$

where $V_{\mathrm{P}}$ and $V_{\mathrm{T}}$ are the potentials of the electron in the field of projectile and target, respectively. Thereby we adapt the three-particle picture, where the effect of all the electrons of the projectile-target system except the active one is incorporated into the potentials. In the semiclassical approximation, the transition amplitude for the electron loss is given by (Dettmann 1971)

$$
a_{f i}=\frac{1}{\mathrm{i} \hbar} \int_{-\infty}^{\infty} \mathrm{d} t\left\langle\Psi_{f}^{-}\left|V_{\mathrm{T}}\right| \psi_{i}^{\mathrm{P}}\right\rangle
$$

where $\psi_{i}^{\mathrm{P}}$ denotes the initial electronic state with energy $\varepsilon_{i}^{\mathrm{P}}$, and $\Psi_{f}^{-}$is the exact solution to (2.1) with the boundary condition of a free electron in the final state. Unlike the case of a bound final state, both potentials $V_{\mathrm{P}}$ and $V_{\mathrm{T}}$ will determine the final electronic wavefunction which thus has a complicated structure. Therefore, we have chosen the prior form for $a_{f i}$ which avoids explicit knowledge of the final state.

According to Faddeev (1961) $\Psi_{f}^{-}$can be written as a sum of wavefunctions which are determined by the set of two coupled equations

$$
\begin{aligned}
& \left|\Psi_{f}^{-}\right\rangle=\left|\psi_{0}\right\rangle+\left|\psi_{1}\right\rangle+\left|\psi_{2}\right\rangle \\
& \left|\psi_{1}\right\rangle=G_{\mathrm{T}} V_{\mathrm{T}}\left|\psi_{0}\right\rangle+G_{\mathrm{T}} V_{\mathrm{T}}\left|\psi_{2}\right\rangle \\
& \left|\psi_{2}\right\rangle=G_{\mathrm{P}} V_{\mathrm{P}}\left|\psi_{0}\right\rangle+G_{\mathrm{P}} V_{\mathrm{P}}\left|\psi_{1}\right\rangle
\end{aligned}
$$


where $\psi_{0}$ is a plane wave and

$$
G_{\mathrm{T}, \mathrm{P}}=\left(\mathrm{i} \hbar \partial / \partial t+\hbar^{2} / 2 m \Delta-V_{\mathrm{T}, \mathrm{P}}-\mathrm{i} \varepsilon\right)^{-1}
$$

is the electron Green's function in the target or projectile field, respectively. From this, one can derive a series expansion of $\Psi_{f}^{-}$

$$
\begin{gathered}
\left|\Psi_{f}^{-}\right\rangle=\left(1+G_{\mathrm{T}} V_{\mathrm{T}}+G_{\mathrm{P}} V_{\mathrm{P}}+G_{\mathrm{T}} V_{\mathrm{T}} G_{\mathrm{P}} V_{\mathrm{P}}+G_{\mathrm{P}} V_{\mathrm{P}} G_{\mathrm{T}} V_{\mathrm{T}}+G_{\mathrm{T}} V_{\mathrm{T}} G_{\mathrm{P}} V_{\mathrm{P}} G_{\mathrm{T}} V_{\mathrm{T}}\right. \\
\left.+G_{\mathrm{P}} V_{\mathrm{P}} G_{\mathrm{T}} V_{\mathrm{T}} G_{\mathrm{P}} V_{\mathrm{P}}+\ldots\right)\left|\psi_{0}\right\rangle
\end{gathered}
$$

which contains the action of the projectile and target field in alternating succession. In order to make the series expansion meaningful we have to assume that the potentials $V_{\mathrm{P}}$ and $V_{\mathrm{T}}$ are screened when $\boldsymbol{r}_{\mathrm{P}}$ or $\boldsymbol{r}_{\mathrm{T}} \rightarrow \infty$, which actually corresponds to a real experimental situation. The choice of a pure Coulomb field is not unproblematic in a higher-order theory due to the non-uniform convergence of a screened Coulomb function to a Coulomb wave (Okubo and Feldman 1960). However, the error introduced by using Coulomb waves in the transition amplitude seems to be small for high projectile velocities as has been shown by Macek and Taulbjerg (1981) in the related case of charge transfer to bound states.

One should note that the series (2.4) is able to describe both a target and a projectile final state, respectively, as it can formally be summed in two ways:

$$
\begin{aligned}
\left|\Psi_{f}^{-}\right\rangle & =\left(1+G_{\mathrm{P}} V_{\mathrm{P}}\right)\left(1+G_{\mathrm{T}} V_{\mathrm{T}} G_{\mathrm{P}} V_{\mathrm{P}}+\ldots\right)\left|\psi_{f}^{\mathrm{T}}\right\rangle \\
& =\left(1+G_{\mathrm{T}} V_{\mathrm{T}}\right)\left(1+G_{\mathrm{P}} V_{\mathrm{P}} G_{\mathrm{T}} V_{\mathrm{T}}+\ldots\right)\left|\psi_{f}^{\mathrm{P}}\right\rangle
\end{aligned}
$$

where use has been made of the definition of a continuum target (projectile) eigenstate

$$
\left|\psi^{\mathrm{T}, \mathrm{P}}\right\rangle=\left(1+G_{\mathrm{T}, \mathrm{P}} V_{\mathrm{T}, \mathrm{P}}\right)\left|\psi_{0}\right\rangle .
$$

However, for a pure Coulomb field the series usually are divergent and one has therefore to be very careful when breaking off the expansion, thereby getting different answers depending on whether one takes $(2.5 a)$ or $(2.5 b)$ as a starting point.

Due to the ambiguity of the final state it is not possible, as in the case of a (fixed) bound final state, to use an expansion in terms of one of the nuclear potentials, an idea on which the impulse approximation for charge transfer is based (Briggs 1977), and which leads to a theory that will be very good for highly asymmetric systems. Instead, we choose the expansion (2.4) which is symmetric in $V_{\mathrm{P}}$ and $V_{\mathrm{T}}$, and which, if higher order terms are included, will not only describe electron emission in symmetric systems $\left(Z_{1} \simeq Z_{2}\right)$, but also both limiting cases $\boldsymbol{k}_{f} \approx 0$ and $\boldsymbol{k}_{f} \simeq m \boldsymbol{v} / \hbar$ ( $\boldsymbol{v}$ is the projectile velocity) even in asymmetric systems. We break the series (2.4) after five terms and call this the semiclassical Faddeev approximation

$$
\left|\Psi_{f}^{-}\right\rangle^{\mathrm{FA}}=\left(1+G_{\mathrm{T}} V_{\mathrm{T}}+G_{\mathrm{P}} V_{\mathrm{P}}+G_{\mathrm{T}} V_{\mathrm{T}} G_{\mathrm{P}} V_{\mathrm{P}}+G_{\mathrm{P}} V_{\mathrm{P}} G_{\mathrm{T}} V_{\mathrm{T}}\right)\left|\psi_{0}\right\rangle
$$

It can be written in a more convenient way by introducing a complete set of plane waves $|\boldsymbol{q}\rangle$ with momentum $\boldsymbol{q}$ and by making use of the eigenstate definition (2.6)

$$
\left|\Psi_{f}^{-}\right\rangle^{\mathrm{FA}}=\int \mathrm{d} \boldsymbol{q}\left|\psi_{\boldsymbol{q}}^{\mathrm{P}}\right\rangle\left\langle\boldsymbol{q} \mid \psi_{f}^{\mathrm{T}}\right\rangle+\int \mathrm{d} \boldsymbol{q}\left|\psi_{\boldsymbol{q}}^{\mathrm{T}}\right\rangle\left\langle\boldsymbol{q} \mid \psi_{f}^{\mathrm{P}}\right\rangle-\left|\psi_{f}^{\mathrm{T}}\right\rangle-\left|\psi_{f}^{\mathrm{P}}\right\rangle+\left|\psi_{0}\right\rangle
$$

The first integral is the sum of the first three and the last term of (2.7) and is equal to the impulse approximation. The second integral is the corresponding expression where the fourth term in (2.7) is used instead of the last. The remaining functions in (2.8) only 
correct for double counting. Unlike the case of a bound final state (JakubassaAmundsen and Amundsen 1980), the continuum wavefunctions $\psi_{q}$ are from the starting point on-shell which means that no approximation is introduced in the step from (2.7) to (2.8).

The transition amplitude for electron loss is obtained by inserting (2.8) into (2.2). From this, the cross section differential in energy $E_{f}$ and angle $\Omega_{f}$ of the emitted electron, as seen in the target frame, follows as an integral over impact parameter $b$

$$
\frac{\mathrm{d}^{2} \sigma}{\mathrm{d} E_{f} \mathrm{~d} \Omega_{f}}=\frac{m k_{f}}{\hbar^{2}} \int \mathrm{d}^{2} b\left|a_{f i}\right|^{2}
$$

which has to be multiplied by the number of electrons in the initial state.

\section{Comparison of the SCFA with other theories}

As the Faddeev theory describes the exact three-body scattering problem it can be used as a starting point to derive the approximations introduced in the literature. Most of these approximations have been developed for the case of target ionisation but they are easily reformulated for the application to electron loss (by interchanging projectile and target), such that they can be extracted from the expansions given in the preceeding section.

The substitution of the exact wavefunction by a projectile eigenstate (corresponding to the first term in the series $(2.5 b)$ ) leads to the first-order Born approximation for ionisation. As has been shown by Drepper and Briggs (1976) in the case of a pure Coulomb field $V_{\mathrm{P}}$, this theory is able to describe the forward peak at $\boldsymbol{k}_{f}=m \boldsymbol{v} / \hbar$ which can be traced back to the divergence of the normalisation constant of $\psi_{f}^{\mathrm{P}}$ when transformed to the target (i.e. laboratory) frame

$N\left(\eta_{\mathrm{P}}\right)=\exp \left(\frac{1}{2} \pi \eta_{\mathrm{P}}\right) \Gamma\left(1+\mathrm{i} \eta_{\mathrm{P}}\right) \simeq\left(2 \pi \eta_{\mathrm{P}}\right)^{1 / 2} \exp \left[-\mathrm{i}\left(\eta_{\mathrm{P}}-\eta_{\mathrm{P}} \ln \eta_{\mathrm{P}}-\frac{1}{4} \pi\right)\right] \quad$ for $\eta_{\mathrm{P}} \rightarrow \infty$

where $\eta_{\mathbf{P}}=\hat{Z}_{1} /\left|\boldsymbol{k}_{f}-m \boldsymbol{v} / \hbar\right|$, and the abbreviation $\hat{Z}=Z e^{2} m / \hbar^{2}$ is used.

For backward emission angles $\vartheta_{f}$ of the electron, especially in the case of heavy targets, this first-order ionisation theory is not sufficient. To take the target field into account, $\Psi_{f}^{-}$has been replaced by a target eigenstate (Jakubassa 1980) which corresponds to the first-order theory for charge transfer. However, in this theory the forward peak cannot be explained as the interaction with the projectile is neglected. It is interesting to note (and shown later on) that if $V_{\mathrm{P}}$ is included in the way indicated in the expansion (2.5a), none of the terms shows a divergence for $\boldsymbol{k}_{f}=m \boldsymbol{v} / \hbar$ which means that a truncation of this series does not give the right answer in this region.

To overcome this difficulty allowance has been made for both a target and a projectile final state by retaining the first term of both series (2.5) which means the inclusion of the three first-order terms in (2.4) (Macek 1970)

$$
\left|\Psi_{f}^{-}\right\rangle^{\mathrm{M}}=\left(1+G_{\mathrm{T}} V_{\mathrm{T}}+G_{\mathrm{P}} V_{\mathrm{P}}\right)\left|\psi_{0}\right\rangle=\left|\psi_{f}^{\mathrm{T}}\right\rangle+\left|\psi_{F}^{\mathrm{P}}\right\rangle-\left|\psi_{0}\right\rangle
$$

Thereby one is able to explain qualitatively the energy distribution of the electrons at all emission angles. However, this theory is limited in its application, as charge transfer cannot properly be described within a first-order theory. As in charge transfer theories to bound states, it is the second-order term in the Born series (corresponding to 
retaining $\left(1+G_{0} V_{\mathrm{P}}\right)\left|\psi_{f}^{\mathrm{T}}\right\rangle$ in the series $\left.(2.5 a)\right)$ that dominates at high impact velocities (Dettmann et al 1974). In order to account for the higher-order contributions, Miraglia and Ponce (1980) suggested the following approximation for the double-differential cross section

$$
\frac{\mathrm{d}^{2} \sigma^{\mathrm{MP}}}{\mathrm{d} E_{f} \mathrm{~d} \Omega_{f}}=(2 \pi)^{3}\left|\psi_{f}^{\mathrm{T}}\left(\boldsymbol{r}_{\mathrm{T}}=0\right)\right|^{2} \frac{\mathrm{d}^{2} \sigma^{\mathrm{ion}}}{\mathrm{d} E_{f} \mathrm{~d} \Omega_{f}}
$$

where $\mathrm{d}^{2} \sigma^{\text {ion }} / \mathrm{d} E_{f} \mathrm{~d} \Omega^{f}$ is the projectile ionisation cross section (as seen in the target frame) in the first Born approximation. Thereby both possible electronic final states are combined in a single expression. This approximation is, however, justified rather by comparison with experiment than by a consistent derivation.

To show this, we proceed with the evaluation of the electron-loss cross section using the SCFA. Corresponding to the five expansion terms in (2.8) we split the transition amplitude (2.2) into five contributions

$$
a_{f i}^{\mathrm{FA}}=\sum_{n=1}^{5} a_{f i}^{(n)}
$$

and consider for the moment only the first one, corresponding to the impulse approximation for charge transfer. Choosing the target system as reference frame, the projectile wavefunction is given by

$$
\psi^{\mathrm{P}}=\psi^{\mathrm{P}}\left(\boldsymbol{r}_{\mathrm{T}}-\boldsymbol{R}\right) \exp \left[(-\mathrm{i} / \hbar)\left(\varepsilon^{\mathrm{P}} t+\frac{1}{2} m v^{2} t-m v \cdot \boldsymbol{r}_{\mathrm{T}}\right)\right]
$$

and the internuclear motion $\boldsymbol{R}=\boldsymbol{b}+\boldsymbol{v} t$ is described by a straight-line path with impact parameter $\boldsymbol{b}$. The transition amplitude follows as (Briggs 1977, Jakubassa-Amundsen and Amundsen 1980)

$$
\begin{array}{rl}
a_{f i}^{(1)}=\frac{1}{\mathrm{i} \hbar} \int_{-\infty}^{\infty} \mathrm{d} & t \int \mathrm{d} \boldsymbol{q} \exp [\mathrm{i}(\Delta E / \hbar-\boldsymbol{q} \cdot \boldsymbol{v}) t] \exp (-\mathrm{i} \boldsymbol{q} \cdot \boldsymbol{b}) \boldsymbol{\varphi}_{f}^{* \mathrm{~T}}(\boldsymbol{q}+m \boldsymbol{v} / \hbar) \\
& \times \int \mathrm{d} \boldsymbol{r}_{\mathrm{P}} \psi_{\boldsymbol{q}}^{* \mathrm{P}}\left(\boldsymbol{r}_{\mathrm{p}}\right) V_{\mathrm{T}}\left(\boldsymbol{r}_{\mathrm{P}}+\boldsymbol{R}\right) \psi_{i}^{\mathrm{P}}\left(\boldsymbol{r}_{\mathrm{p}}\right)
\end{array}
$$

where $\Delta E=E_{f}-\varepsilon_{i}^{\mathrm{P}}-\frac{1}{2} m v^{2}$ is the energy difference and $\varphi_{f}^{* \mathrm{~T}}(\boldsymbol{q}+m \boldsymbol{v} / \hbar)$ the Fourier transform of the target continuum state. It is convenient to introduce the Fourier transform of the target field, which for a screened Coulomb potential is given by

$$
V_{\mathrm{T}}\left(\boldsymbol{r}_{\mathrm{T}}\right)=-\frac{Z_{2} e^{2}}{r_{\mathrm{T}}} \exp \left(-\alpha r_{\mathrm{T}}\right)=-\frac{Z_{2} e^{2}}{2 \pi^{2}} \int \frac{\mathrm{d} s}{s^{2}+\alpha^{2}} \exp \left(\mathrm{i} s \cdot \boldsymbol{r}_{\mathrm{T}}\right)
$$

and to make the substitution $q_{0}=q-s$. Then the time integral leads to a $\delta$ function, and the integration over impact parameter $(2.9)$ is easily performed by means of the relation

$$
\begin{aligned}
\int \mathrm{d}^{2} \boldsymbol{b} \mid \int \mathrm{d} \boldsymbol{q}_{0} & \left.\delta\left(\Delta E / \hbar-\boldsymbol{q}_{0} \cdot \boldsymbol{v}\right) f\left(\boldsymbol{q}_{0}\right) \exp \left(-\mathrm{i} \boldsymbol{q}_{0} \cdot \boldsymbol{b}\right)\right|^{2} \\
= & \frac{(2 \pi)^{2}}{v} \int \mathrm{d} \boldsymbol{q}_{0} \delta\left(\Delta E / \hbar-\boldsymbol{q}_{0} \cdot \boldsymbol{v}\right)\left|f\left(\boldsymbol{q}_{0}\right)\right|^{2}
\end{aligned}
$$

which holds because $b$ is perpendicular to $v$. For a pure Coulomb target field $(\alpha=0)$, 
the contribution of $a_{f i}^{(1)}$ to the cross section is thus

$$
\begin{aligned}
\frac{\mathrm{d}^{2} \sigma^{(1)}}{\mathrm{d} E_{f} \mathrm{~d} \Omega_{f}}=\frac{4 \hat{Z}_{2}^{2} k_{f}}{m v} \int \mathrm{d} \boldsymbol{q}_{0} \delta\left(\Delta E / \hbar-\boldsymbol{q}_{0} \cdot \boldsymbol{v}\right) \\
\quad \times\left|\int \frac{\mathrm{d} s}{s^{2}} \varphi_{f}^{* \mathrm{~T}}\left(\boldsymbol{q}_{0}+s+m \boldsymbol{v} / \hbar\right)\left\langle\psi_{\boldsymbol{q}_{0}+\boldsymbol{s}}^{\mathrm{P}}\left|\exp \left(\mathrm{i} s \cdot \boldsymbol{r}_{\mathrm{p}}\right)\right| \psi_{i}^{\mathrm{P}}\right\rangle\right|^{2} .
\end{aligned}
$$

From this, the Miraglia-Ponce approximation can be deduced by noting that the Fourier transform of the target Coulomb wave (Bethe and Salpeter 1957)

$$
\begin{aligned}
& \varphi_{f}^{* \mathrm{~T}}(\boldsymbol{k})=\frac{1}{\pi^{2}} \exp \left(\frac{1}{2} \pi \eta_{f}\right) \Gamma\left(1-\mathrm{i} \eta_{f}\right) \lim _{\varepsilon \rightarrow 0}\left(\eta_{f}\left(k_{f}+\mathrm{i} \varepsilon\right) \frac{\left[k^{2}-\left(k_{f}+i \varepsilon\right)^{2}\right]^{-\mathrm{i} \eta_{f}-1}}{\left[\left(\boldsymbol{k}-\boldsymbol{k}_{f}\right)^{2}+\varepsilon^{2}\right]^{1-\mathrm{i} \eta_{f}}}\right. \\
&\left.+\left(1-\mathrm{i} \eta_{f}\right) \varepsilon \frac{\left[k^{2}-\left(k_{f}+\mathrm{i} \varepsilon\right)^{2}\right]^{-\mathrm{i} \eta_{f}}}{\left[\left(\boldsymbol{k}-\boldsymbol{k}_{f}\right)^{2}+\varepsilon^{2}\right]^{2-\mathrm{i} \eta_{f}}}\right)
\end{aligned}
$$

where $\eta_{f}=\hat{Z}_{2} / k_{f}$, is strongly peaked at

$$
\boldsymbol{k} \equiv \boldsymbol{q}_{0}+\boldsymbol{s}+m \boldsymbol{v} / \hbar=\boldsymbol{k}_{f}
$$

(the second term becomes $\boldsymbol{\delta}\left(\boldsymbol{k}-\boldsymbol{k}_{f}\right)$ for $\eta_{f}=0$ and vanishes otherwise) such that one may approximate (3.9) by taking everything but $\varphi_{f}^{* \mathrm{~T}}$ outside the $s$ integral at the peaking value. The $s$ integral then simply yields the target wavefunction at $\boldsymbol{r}_{\mathrm{T}}=0$, and the remainder can be shown to lead to the first-order Born approximation for ionisation $\left(\boldsymbol{q}_{0}+\boldsymbol{s}=\boldsymbol{k}_{f}-\boldsymbol{m} \boldsymbol{v} / \hbar\right.$ is the electron momentum in the projectile frame), such that one arrives at (3.3).

This approximation shows the forward divergence at $\boldsymbol{k}_{f}=\boldsymbol{m} \boldsymbol{v} / \hbar$ as the normalisation constant of the projectile (final) state factors out. However, the peaking approximation leading to (3.3) is not justified around $\boldsymbol{k}_{f}=m \boldsymbol{v} / \hbar$ because the normalisation $N(\eta)$ of $\psi_{q_{0}+s}^{\mathrm{P}}$ in (3.9) with $\eta=\hat{Z}_{1} /\left|q_{0}+s\right|$, is according to (3.1) strongly oscillating at $s=-\boldsymbol{q}_{0}$ and thus must be kept inside the $s$ integral when the peaking approximation is performed.

The result of a more careful evaluation of (3.9) is that it has a finite value at $\boldsymbol{k}_{f}=m \boldsymbol{v} / \hbar$ and thus no forward divergence. This can be seen by making the substitution $s_{0}=q_{0}+s+m \boldsymbol{v} / \hbar-\boldsymbol{k}_{f}$ in the $s$ integral together with the peaking approximation for the slowly varying functions in $s$. When spherical coordinates are used, the $s$ integral then reduces for $\boldsymbol{k}_{f}=m \boldsymbol{v} / \hbar$ to

$$
\begin{aligned}
& \int \mathrm{d} s_{0} \varphi_{f}^{* \mathrm{~T}}\left(\boldsymbol{s}_{0}+\boldsymbol{k}_{f}\right) \exp \left(\frac{1}{2} \pi \eta\right) \Gamma(1-\mathrm{i} \eta) \\
&=\frac{1}{\pi \mathrm{i}} \exp \left(\frac{1}{2} \pi \eta_{f}\right) \Gamma\left(1-\mathrm{i} \eta_{f}\right) \int_{0}^{\infty} \mathrm{d} s_{0} s_{0}^{-1+\mathrm{i} \eta_{f}} \exp \left[\pi \hat{Z}_{1} /\left(2 s_{0}\right)\right] \\
& \times \Gamma\left(1-\mathrm{i} \hat{Z}_{1} / s_{0}\right)\left[\left(s_{0}-2 k_{f}\right)^{-\mathrm{i} \eta_{f}}-\left(s_{0}+2 k_{f}\right)^{-\mathrm{i} \eta_{f}}\right] .
\end{aligned}
$$

It is then easy to verify that (3.11) is finite. In complete analogy it can be shown that none of the higher terms of the series $(2.5 a)$-when going beyond the impulse approximation-shows a divergence, as any appearance of a projectile eigenstate, indicated by the term $G_{\mathrm{P}} V_{\mathrm{P}}$, is combined with an integral over momentum which cancels the divergence of the normalisation factor. From this it follows that a factorisation of the type (3.3), although valid if $\boldsymbol{k}_{f}$ is far apart from $m \boldsymbol{v} / \hbar$ where the normalisation 
is a smooth function, cannot be considered as an appropriate theory for arbitrary electron momenta.

\section{The forward peak and numerical calculations}

In this section we apply the semiclassical Faddeev approximation to the ionisation of hydrogen by a heavy target. This means that the potential between the active electron and the projectile is purely Coulombic, while the target field is screened by the target electrons. We use the potential (3.7) with the screening constant $\alpha=2.74 m e^{2} / \hbar$ (for $\mathrm{Ne}$ ) which is the dominant term in a fit to a Hartree-Fock potential (Strand and Bonham 1964). For the target functions $\psi^{\mathrm{T}}$ one should take the corresponding eigenfunctions. While for lower impact energies it is important to use the exact eigenfunctions which allow for resonant electronic excitations in the target (Duncan and Menendez 1979, Jakubassa 1980) one may think of using hydrogenic wavefunctions when the impact velocity becomes as large as the target $\mathrm{K}$-shell orbiting velocity. The influence on the energy distribution is small anyhow, and the difference between (3.6) and a pure Coulomb field affects mainly the absolute value of the cross section. Thus we use Coulomb waves for $\psi^{\mathrm{T}}$ in the following which simplifies the calculation considerably.

It remains to calculate the amplitudes $a_{f i}^{(2)}-a_{f i}^{(5)}$ and add them coherently to the first one. The second term in the series (2.8) leads to the transition amplitude

$$
\begin{aligned}
& a_{f i}^{(2)}=\frac{1}{\mathrm{i} \hbar} \int_{-\infty}^{\infty} \mathrm{d} t \int \mathrm{d} \boldsymbol{q} \exp \left[(\mathrm{i} / \hbar)\left(\varepsilon_{f}^{\mathrm{P}}-\varepsilon_{i}^{\mathrm{P}}-m v^{2}+\hbar \boldsymbol{q} \cdot \boldsymbol{v}\right) t\right] \exp (\mathrm{i} \boldsymbol{q} \cdot \boldsymbol{b}) \varphi_{f}^{* \mathrm{P}}(\boldsymbol{q}-m \boldsymbol{v} / \hbar) \\
& \times \int \mathrm{d} \boldsymbol{r}_{\mathrm{T}} \psi_{\boldsymbol{q}}^{* \mathrm{~T}}\left(\boldsymbol{r}_{\mathrm{T}}\right) V_{\mathrm{T}}\left(\boldsymbol{r}_{\mathrm{T}}\right) \psi_{i}^{\mathrm{P}}\left(\boldsymbol{r}_{\mathrm{T}}-\boldsymbol{R}\right) \exp \left(\mathrm{i} m \boldsymbol{v} \cdot \boldsymbol{r}_{\mathrm{T}} / \hbar\right)
\end{aligned}
$$

For further evaluation we introduce $\varphi_{i}^{\mathrm{P}}(s)$, the Fourier transform of the projectile bound state, which equals $2^{3 / 2} \hat{Z}_{1}^{5 / 2}\left(\hat{Z}_{1}^{2}+s^{2}\right)^{-2} / \pi$ for a 1 s state. Then the time integral can be performed and leads to a $\delta$ function as before. However, as

$$
\varepsilon_{f}^{\mathrm{P}}=\frac{1}{2} m\left(\boldsymbol{k}_{f}-\boldsymbol{v}\right)^{2}=E_{f}+\frac{1}{2} m v^{2}-\hbar \boldsymbol{k}_{f} \cdot \boldsymbol{v}
$$

is now the final electron energy in the projectile frame, the argument in the $\delta$ function looks different from the one in $a_{f i}^{(1)}$. In order to add the amplitudes coherently it is convenient to make a coordinate transformation from $\boldsymbol{q}$ to $\boldsymbol{q}_{0}=-\boldsymbol{q}+\boldsymbol{s}+\boldsymbol{k}_{f}$ such that the $\delta$ functions become equal. On the other hand one thereby introduces an impact parameter dependent phase, $\exp \left(i \boldsymbol{k}_{f} \cdot \boldsymbol{b}\right)$, which is not present in $a_{f i}^{(1)}$. This phase originates from the transformation of a projectile continuum state into the target frame if the prescription (3.5) is used. As this phase is constant in space and time one may as well include $\exp \left(\mathrm{i}_{\boldsymbol{f}} \cdot \boldsymbol{b}\right)$ in the transformation operator for the final continuum projectile state without affecting the property that the transformed wavefunction is a solution of the corresponding Schrödinger equation. Then $\exp \left(\mathrm{i}_{f} \cdot \boldsymbol{b}\right)$ disappears from the transition probability in agreement with a full quantum mechanical description.

The remaining amplitudes $a_{f i}^{(3)}-a_{f i}^{(5)}$ are obtained in a similar way. The integration over impact parameter leads to

$$
\frac{\mathrm{d}^{2} \sigma^{\mathrm{FA}}}{\mathrm{d} E_{f} \mathrm{~d} \Omega_{f}}=\frac{4 \hat{Z}_{2}^{2} k_{f}}{m v} \int \mathrm{d} \boldsymbol{q}_{0} \delta\left(\Delta E / \hbar-\boldsymbol{q}_{0} \cdot v\right)\left|\sum_{n=1}^{5} A_{n}\right|^{2}
$$


with

$$
\begin{gathered}
A_{1}=-\int \frac{\mathrm{d} s}{s^{2}+\alpha^{2}} \varphi_{f}^{* \mathrm{~T}}\left(\boldsymbol{q}_{0}+s+m \boldsymbol{v} / \hbar\right)\left\langle\psi_{\boldsymbol{q}_{0}+s}^{\mathrm{P}}\left|\exp \left(\mathrm{is} \cdot \boldsymbol{r}_{\mathrm{P}}\right)\right| \psi_{i}^{\mathrm{P}}\right\rangle \\
A_{2}=-\left(\frac{1}{2} \pi\right)^{1 / 2} \int \mathrm{d} \boldsymbol{s} \varphi_{i}^{\mathrm{P}}(\boldsymbol{s}) \varphi_{f}^{* \mathrm{P}}\left(-\boldsymbol{q}_{0}+\boldsymbol{s}+\boldsymbol{k}_{f}-m \boldsymbol{v} / \hbar\right) \\
\times\left\langle\psi_{-\boldsymbol{q}_{0}+\boldsymbol{s}+\boldsymbol{k}_{f}}^{\mathrm{T}} \exp \left(-\alpha r_{\mathrm{T}}\right) / r_{\mathrm{T}} \mid \exp \left[\mathrm{i}(\boldsymbol{s}+m \boldsymbol{v} / \hbar) \boldsymbol{r}_{\mathrm{T}}\right]\right\rangle \\
A_{3}=\left(\frac{1}{2} \pi\right)^{1 / 2} \varphi_{i}^{\mathrm{P}}\left(\boldsymbol{q}_{0}\right)\left\langle\psi_{f}^{\mathrm{T}}\left|\exp \left(-\alpha r_{\mathrm{T}}\right) / r_{\mathrm{T}}\right| \exp \left[\mathrm{i}\left(\boldsymbol{q}_{0}+m \boldsymbol{v} / \hbar\right) \boldsymbol{r}_{\mathrm{T}}\right]\right\rangle \\
A_{4}=\frac{1}{\left(\boldsymbol{q}_{0}-\boldsymbol{k}_{f}+m \boldsymbol{v} / \hbar\right)^{2}+\alpha^{2}}\left\langle\psi_{f}^{\mathrm{P}}\left|\exp \left[\mathrm{i}\left(-\boldsymbol{q}_{0}+\boldsymbol{k}_{f}-m \boldsymbol{v} / \hbar\right) \boldsymbol{r}_{\mathrm{P}}\right]\right| \psi_{i}^{\mathrm{P}}\right\rangle \\
A_{5}=-\frac{1}{\left(\boldsymbol{q}_{0}-\boldsymbol{k}_{f}+m \boldsymbol{v} / \hbar\right)^{2}+\alpha^{2}} \varphi_{i}^{\mathrm{P}}\left(\boldsymbol{q}_{0}\right) .
\end{gathered}
$$

Our aim is to study the influence of the target field on the energy distribution of the ejected electrons in a region where the projectile field is also important. We therefore choose the emission direction $\boldsymbol{k}_{f}$ of the electrons to be parallel to $v$, corresponding to $\vartheta_{f}=0$. This also means a considerable simplification of the calculations, such that $(4.2)$ can be evaluated without any further approximation.

When hydrogenic wavefunctions are used, all matrix elements can be evaluated analytically (Nordsieck 1954). For a 1 s initial state, one obtains (McDowell and Coleman 1970)

$$
\left\langle\psi_{\boldsymbol{k}}^{\mathrm{P}}\left|\exp \left(\mathrm{i} \boldsymbol{q} \cdot \boldsymbol{r}_{\mathrm{P}}\right)\right| \psi_{i}^{\mathrm{P}}\right\rangle=\left(2^{3 / 2} \hat{Z}_{1}^{5 / 2} / \pi\right) M_{1}(k, q,|\boldsymbol{q}-\boldsymbol{k}|)
$$

$M_{1}(k, q,|q-k|)$

$$
\begin{aligned}
= & \exp \left(\frac{1}{2} \pi \eta_{1}\right) \Gamma\left(1-\mathrm{i} \eta_{1}\right) \frac{\left[q^{2}-\left(k+\mathrm{i} \hat{Z}_{1}\right)^{2}\right]^{-\mathrm{i} \eta_{1}}}{\left[\hat{Z}_{1}^{2}+(\boldsymbol{q}-\boldsymbol{k})^{2}\right]^{2-\mathrm{i} \eta_{1}}} \\
& \times\left(\left(1+\mathrm{i} \eta_{1}\right) \frac{\hat{Z}_{1}^{2}+(\boldsymbol{q}-\boldsymbol{k})^{2}}{q^{2}-\left(k+\mathrm{i} \hat{\boldsymbol{Z}}_{1}\right)^{2}}+1-\mathrm{i} \eta_{1}\right)
\end{aligned}
$$

with $\eta_{1}=\hat{Z}_{1} / k$, and

$$
\begin{aligned}
& \left\langle\psi_{\boldsymbol{k}}^{\mathrm{T}}\left|\exp \left(-\alpha r_{\mathrm{T}}\right) / r_{\mathrm{T}}\right| \exp \left(\mathrm{i} \boldsymbol{q} \cdot \boldsymbol{r}_{\mathrm{T}}\right)\right\rangle=(2 / \pi)^{1 / 2} \boldsymbol{M}_{\mathbf{2}}(k, q,|\boldsymbol{q}-\boldsymbol{k}|) \\
& M_{2}(k, q,|\boldsymbol{q}-\boldsymbol{k}|)=\exp \left(\frac{1}{2} \pi \eta_{2}\right) \Gamma\left(1-\mathrm{i} \eta_{2}\right) \frac{\left[q^{2}-(k+\mathrm{i} \alpha)^{2}\right]^{-\mathrm{i} \eta_{2}}}{\left[(\boldsymbol{q}-\boldsymbol{k})^{2}+\alpha^{2}\right]^{1-\mathrm{i} \eta_{2}}}
\end{aligned}
$$

with $\eta_{2}=\hat{Z}_{2} / k$.

For further evaluation we introduce the variable $\boldsymbol{q}_{1}=-\boldsymbol{q}_{0}+\boldsymbol{k}_{f}-m \boldsymbol{v} / \hbar$ and make the substitution $s_{0}=s-q_{1}$ in $A_{1}$ as before, while in $A_{2}$ we change variables according to

$$
s_{0}=s+q_{1}-k_{f}+m v / \hbar
$$

in order to cope numerically with the divergence in the Fourier transformed Coulomb wave. Then $A_{1}$ and $A_{2}$ change into

$$
\begin{aligned}
A_{1}=-\left(2^{3 / 2} \hat{Z}_{1}^{5 / 2} / \pi\right) \int \frac{\mathrm{d} s_{0}}{\left(s_{0}+q_{1}\right)^{2}+\alpha^{2}} \varphi_{f}^{* \mathrm{~T}}\left(s_{0}+\boldsymbol{k}_{f}\right) \\
\quad \times M_{1}\left(\left|s_{0}+\boldsymbol{k}_{f}-m \boldsymbol{v} / \hbar\right|,\left|s_{0}+\boldsymbol{q}_{1}\right|,\left|\boldsymbol{q}_{1}+m \boldsymbol{v} / \hbar-\boldsymbol{k}_{f}\right|\right)
\end{aligned}
$$


$A_{2}=-\int \mathrm{d} s_{0} \varphi_{i}^{\mathrm{P}}\left(\boldsymbol{s}_{0}-\boldsymbol{q}_{1}+\boldsymbol{k}_{f}-m \boldsymbol{v} / \hbar\right) \varphi_{f}^{* \mathrm{P}}\left(\boldsymbol{s}_{0}+\boldsymbol{k}_{f}-m \boldsymbol{v} / \hbar\right) M_{2}\left(\left|\boldsymbol{s}_{0}+\boldsymbol{k}_{f}\right|,\left|\boldsymbol{s}_{0}-\boldsymbol{q}_{1}+\boldsymbol{k}_{f}\right|, q_{1}\right)$

and the argument of the energy conserving $\delta$ function turns into

$$
\hbar\left(\boldsymbol{k}_{f}-m \boldsymbol{v} / \hbar\right)^{2} /(2 m)-\varepsilon_{i}^{\mathrm{P}} / \hbar+\boldsymbol{q}_{1} \cdot \boldsymbol{v} .
$$

Note that the final momentum of the projectile state $\varphi_{f}^{* \mathrm{P}}$ is $\boldsymbol{k}_{f}-m \boldsymbol{v} / \hbar$, while it is $\boldsymbol{k}_{f}$ for the target state $\varphi_{f}^{* \mathrm{~T}}$. As we have chosen $\boldsymbol{k}_{f}$ parallel to $v, M_{1}$ and $M_{2}$ in (4.4) depend only on the angles $\vartheta_{q_{1}}, \vartheta_{s_{0}}$ (with respect to the $z$ direction $v$ ) and $\vartheta_{s q}$ (which is the angle between $s_{0}$ and $q_{1}$ ). By decomposing

$$
\cos \vartheta_{s q}=\cos \vartheta_{s_{0}} \cos \vartheta_{q_{1}}+\sin \vartheta_{s_{0}} \sin \vartheta_{q_{1}} \cos \varphi_{s_{0}}
$$

the integrand is seen to be a function of $\vartheta_{s_{0}}, \varphi_{s_{0}}$ and $\vartheta_{q_{1}}$, but independent of $\varphi_{q_{1}}$. While the integral over $\vartheta_{q_{1}}$ can be performed by means of the $\delta$ function in (4.2), there remain thus four integrals to be evaluated numerically. With the use of (4.3), (4.4) and (3.10) the differential cross section (4.2) reduces to

$$
\begin{aligned}
\frac{\mathrm{d}^{2} \sigma^{\mathrm{FA}}}{\mathrm{d} E_{f} \mathrm{~d} \Omega_{f}}=\frac{64}{Z_{2}^{2}} \hat{Z}_{1}^{5} k_{f} & \int_{q_{\min }}^{\infty} q_{1} \mathrm{~d} q_{1} \\
& \times \lim _{\varepsilon \rightarrow 0} \mid-\left(2 \hat{Z}_{2} / \pi^{2}\right) \mathrm{e}^{\pi \eta_{f} / 2} \Gamma\left(1-\mathrm{i} \eta_{f}\right) \int_{0}^{\infty} \mathrm{d} s_{0}\left(s_{0}+\varepsilon^{2} / s_{0}\right)^{-1+\mathrm{i} \eta_{f}} \\
& \times \int_{-1}^{1} \mathrm{~d} x\left(s_{0}+2 k_{f} x-2 \mathrm{i} k_{f} \varepsilon / s_{0}+\varepsilon^{2} / s_{0}\right)^{-\mathrm{i} \eta_{f}-1} \\
& \times \int_{0}^{\pi} \mathrm{d} \varphi_{s_{0}} \frac{1}{\left(s_{0}+\boldsymbol{q}_{1}\right)^{2}+\alpha^{2}} M_{1}\left(\left|s_{0}+\boldsymbol{k}_{f}-m \boldsymbol{v} / \hbar\right|,\left|s_{0}+\boldsymbol{q}_{1}\right|,\left|\boldsymbol{q}_{1}-\boldsymbol{k}_{f}+m \boldsymbol{v} / \hbar\right|\right) \\
& -\left(2 \hat{Z}_{1} / \pi^{2}\right) \mathrm{e}^{\pi \eta_{\mathrm{p}} / 2} \Gamma\left(1-\mathrm{i} \eta_{\mathrm{P}}\right) \int_{0}^{\infty} \mathrm{d} s_{0}\left(s_{0}+\varepsilon^{2} / s_{0}\right)^{-1+\mathrm{i} \eta_{\mathrm{p}}} \\
& \times \int_{-1}^{1} \mathrm{~d} x\left[s_{0}+2\left(k_{f}-m v / \hbar\right) x-2 \mathrm{i}\left|k_{f}-m v / \hbar\right| \varepsilon / s_{0}+\varepsilon^{2} / s_{0}\right]^{-\mathrm{i} \eta_{\mathrm{p}}-1} \\
& \times \int_{0}^{\pi} \mathrm{d} \varphi_{s_{0}} \frac{1}{\left[\hat{Z}_{1}^{2}+\left(s_{0}-\boldsymbol{q}_{1}+\boldsymbol{k}_{f}-m \boldsymbol{v} / \hbar\right)^{2}\right]^{2}} \boldsymbol{M}_{2}\left(\left|\boldsymbol{s}_{0}+\boldsymbol{k}_{f}\right|,\left|\boldsymbol{s}_{0}-\boldsymbol{q}_{1}+\boldsymbol{k}_{f}\right|, q_{1}\right) \\
& +\frac{1}{\left[\hat{Z}_{1}^{2}+\left(\boldsymbol{q}_{1}-\boldsymbol{k}_{f}+m \boldsymbol{v} / \hbar\right)^{2}\right]^{2}}\left(\boldsymbol{M}_{2}\left(k_{f},\left|\boldsymbol{q}_{1}-\boldsymbol{k}_{f}\right|, q_{1}\right)-\frac{1}{q_{1}^{2}+\alpha^{2}}\right) \\
& +\left.\frac{1}{q_{1}^{2}+\alpha^{2}} M_{1}\left(\left|\boldsymbol{k}_{f}-m \boldsymbol{v} / \hbar\right|, q_{1},\left|\boldsymbol{q}_{1}-\boldsymbol{k}_{f}+m \boldsymbol{v} / \hbar\right|\right)\right|^{2}
\end{aligned}
$$

In this expression we have introduced the definitions

$$
q_{\min }=-q_{1} \cos \vartheta_{q_{1}}=\left[\hbar^{2}\left(k_{f}-m v / \hbar\right)^{2} /(2 m)-\varepsilon_{i}^{\mathrm{P}}\right] /(\hbar v),
$$

$x=\cos \vartheta_{s_{0}}, \eta_{f}=\hat{Z}_{2} / k_{f}$ and $\eta_{\mathrm{p}}=\hat{Z}_{1} /\left|k_{f}-m v / \hbar\right|$. We recall that $(4.5)$ is only valid for $\boldsymbol{k}_{f}=k_{f} v / v$. For numerical reasons, it is convenient to calculate (4.5) with a small, but finite $\varepsilon$. This is, however, not necessary for convergence reasons (as demonstrated in $\S 3$ ), and one only needs an infinitesimal $\varepsilon$ to determine the analytical behaviour when passing the singularities (Nordsieck 1954). But with the choice of $\varepsilon=+0$ one would have to extract the singularity at $s_{0}=0$ by splitting the integrand and doing the critical 
part analytically. We used $\varepsilon=0.01$ which implies an error of less than one per cent compared to the result with $\varepsilon=+0$. Due to the screening constant $\alpha$, the $\varphi_{s_{0}}$ integral requires only a small step number, while the integrals over $x$ and $s_{0}$ have to be done with high precision because of several (integrable) singularities. We estimate the accuracy of our calculations to be about $5-10 \%$.

As an example, we have chosen the ionisation of hydrogen in collisions with $\mathrm{Ne}$ $\left(Z_{2}=10\right)$ at the impact velocity $v=10 e^{2} / \hbar(E=2.5 \mathrm{MeV})$ which is equal to the $\mathrm{Ne}$ $\mathrm{K}$-shell velocity. We calculated the various contributions to the double-differential cross section separately to study the influence of both the projectile and the target field. Figure 1 shows the electron spectrum calculated from equation (3.9), that is, where the electron is taken to be in a target final state. As shown before, the differential cross section is finite at the resonance $k_{f}=m v / \hbar$ and the narrow oscillations in that region can be traced back to the influence of the strongly oscillating normalisation constant $N(\eta)$ of the projectile intermediate state when $\eta \rightarrow \infty$. Oscillations of this kind usually do not appear in the first or second Born approximation, as the phase of the wavefunctions drops out of the transition probability.

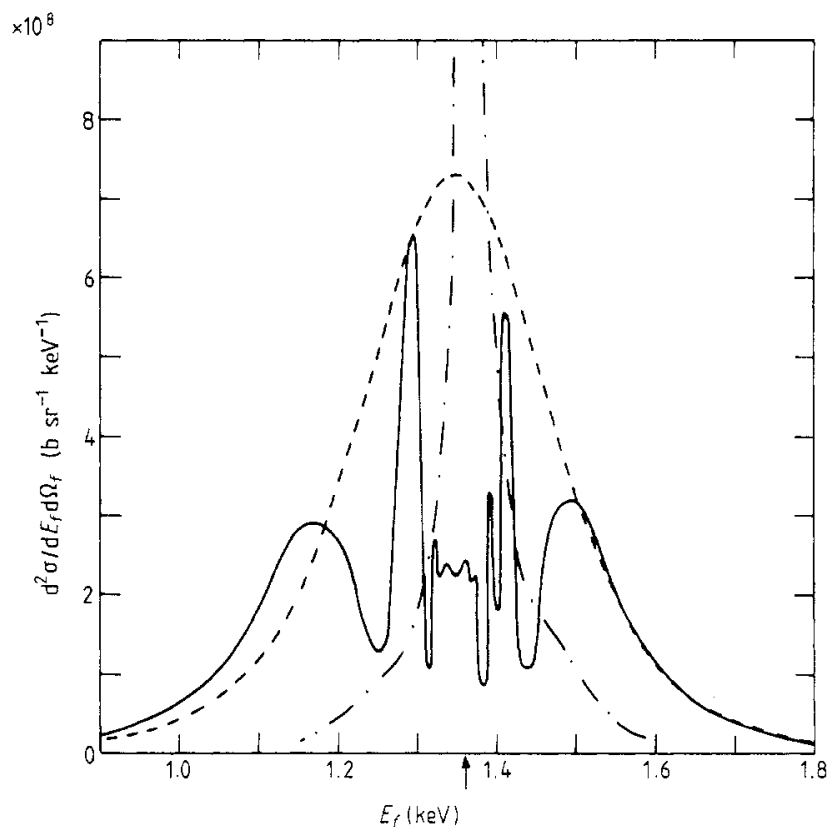

Figure 1. Energy distribution of projectile electrons emitted in $2.5 \mathrm{MeV}(\mathrm{H}, \mathrm{Ne})$ collisions at zero emission angle. The full curve includes only the charge transfer term $A_{1}$ in (4.2) while the chain curve includes only $A_{2}$. The broken curve is the (normalised) Compton profile (4.6) of the projectile. The arrow denotes the energy $\frac{1}{2} m v^{2}$.

The width of the energy distribution at zero emission angle is very similar to the width at larger angles $\vartheta_{f}$ if the electron is captured into a target continuum state. It is roughly given by the momentum distribution of the initial state (Burch et al 1973) which can be expressed by means of the Compton profile (Jakubassa 1980)

$$
J_{i}\left(q_{0 z}\right)=\frac{8 \hat{Z}_{1}^{5}}{3 \pi}\left(q_{0 z}^{2}+\hat{Z}_{1}^{2}\right)^{-3} \quad q_{0 z}=\Delta E /(\hbar v)
$$


for a hydrogenic 1 s state. It is shown in figure 1 for comparison (normalised to the maximum of the energy distribution from (3.9)).

Also shown in figure 1 is the contribution of (4.1) to the differential cross section, where the electron remains in a projectile eigenstate. This term contains the first Born approximation, as well as the second one, because the target field is included to all orders in the intermediate state. As the projectile normalisation factors out, one obtains the well known divergence at $k_{f}=m v / \hbar$. This leads to the narrow forward peak with a much smaller width than for larger emission angles (Duncan and Menendez 1979). The energy distribution shows an asymmetry which is already present in the first Born approximation (Day 1980) but which is enhanced when higher-order terms are included.

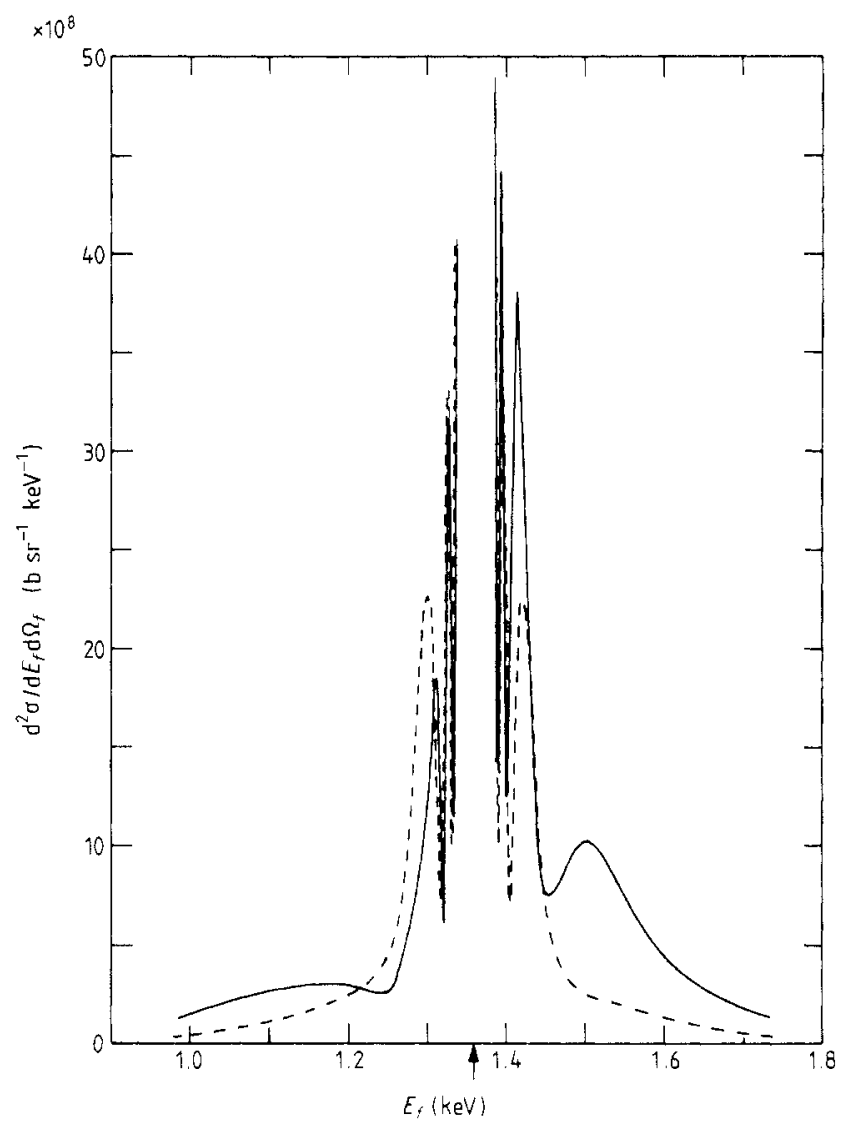

Figure 2. Energy distribution of projectile electrons emitted in $2.5 \mathrm{MeV}(\mathrm{H}, \mathrm{Ne})$ collisions at $\vartheta_{f}=0$. The full curve denotes the complete SCFA (4.2) while the broken curve is the first-order contribution (including only $A_{3}-A_{5}$ in (4.2)). The arrow marks the energy $\frac{1}{2} m v^{2}$.

Figure 2 shows the result of the full calculation (4.5) where the amplitudes (3.6) and (4.1) are added coherently (and double counting is corrected for). As this theory includes both the projectile and the target state as possible final states of the electron the forward cusp is retained, as well as the narrow oscillations on the wings from the oscillating phase of the projectile function. At electron energies slightly off the peak 
(around 1.15 and $1.5 \mathrm{keV}$ ) the structure resulting from the capture into target continuum states survives, and the asymmetry of the energy distribution is strongly increased. For comparison figure 2 shows also the first-order approximation of Macek (1970) which is obtained from (4.2) when only the terms $A_{3}-A_{5}$ are included. There is some discrepancy between the two theories, especially at larger electron energies, which indicates the importance of including the target field to higher orders.

One should note that the narrow oscillations on the wings of the cusp will be washed out in an experimental situation due to the finite angular (or energy) resolution of the detector, which also leads to a finite peak height. In order to compare with experiment one should therefore integrate over the experimental acceptance angle (or over the energy resolution, whatever has a larger uncertainty (Briggs and Day 1980)). However, the broad structures on the outer wings presumably will survive.

\section{Concluding remarks}

We have formulated a one-electron theory for electron loss in fast collisions which includes the higher-order contributions of the projectile and the target potential in a symmetric way and which should thus be valid for arbitrary energies and directions of the emitted electron. In order to study the influence of both the target and the projectile field we applied it to the ejection of electrons from hydrogen by a heavy target in the forward direction. When hydrogenic wavefunctions are used the cross section can be evaluated in this case (and also for $180^{\circ}$ emission angle) without any further approximation. Calculations performed for the $(\mathrm{H}, \mathrm{Ne})$ system support the idea that at low electron momenta with respect to the projectile frame the electrons are ejected into a projectile continuum state, while for larger $\left|k_{f}-m v / \hbar\right|$ the electrons will be captured into a target continuum state. The limit between the two regions is of the order of $\left|\hbar k_{f} / m-v\right| / v \simeq Z_{1} / Z_{2}$ which means that the heavier the target, the more the wings of the forward peak will be influenced by the target. This shows clearly that a first-order theory, until now applied mainly to $(\mathrm{He}, \mathrm{He})$ and $(\mathrm{He}, \mathrm{H})$ collisions, is no longer sufficient for asymmetric systems with $Z_{1} / Z_{2} \ll 1$. It might be interesting to look experimentally for structures on the wings of the forward peak (at high impact velocities where the capture of target electrons into the projectile continuum can be neglected) as a function of the target atom in asymmetric collisions.

\section{Acknowledgment}

I should like to thank K Taulbjerg for critical comments, and the theory group in Oslo for discussions and for their hospitality during my stay in Norway. A partial support by NORDITA and GSI Darmstadt is gratefully acknowledged.

\section{References}

Bethe H A and Salpeter E E 1957 Handbuch der Physik vol 35, ed S Flügge (Berlin: Springer) p 126

Briggs J S 1977 J. Phys. B: At. Mol. Phys. 103075

1980 J. Phys. B: At. Mol. Phys. 13 L717

Briggs J S and Day M H 1980 J. Phys. B: At. Mol. Phys. 134797 
Burch D, Wiemann H and Ingalls W B 1973 Phys. Rev. Lett. 30823

Day M H 1980 J. Phys. B: At. Mol. Phys. 13 L65

Dettmann K 1971 Springer Tracts in Modern Physics vol 58 (Berlin: Springer) p 119

Dettmann K, Harrison K G and Lucas M W 1974 J. Phys. B: At. Mol. Phys. 7269

Drepper F and Briggs J S 1976 J. Phys. B: At. Mol. Phys. 92063

Duncan M M and Menendez M G 1979 Phys. Rev. A 1949

Faddeev L D 1961 Sov. Phys.-JETP 121014

Garibotti C R and Miraglia J E 1980 Phys. Rev. A 21572

Jakubassa D H 1980 J. Phys. B: At. Mol. Phys. 132099

Jakubassa-Amundsen D H and Amundsen P A 1980 Z. Phys. A 297203

McDowell M R C and Coleman J P 1970 Introduction to the Theory of Ion-Atom Collisions (Amsterdam: North-Holland) ch 6-8

Macek J 1970 Phys. Rev. A 1235

Macek J and Taulbjerg K 1981 Phys. Rev. Lett. 46170

Miraglia J E and Ponce V H 1980 J. Phys. B: At. Mol. Phys. 131195

Nordsieck A 1954 Phys. Rev. 93785

Okubo S and Feldman D 1960 Phys. Rev. 117292

Rudd M E, Sautter C A and Bailey C L 1966 Phys. Rev. 15120

Salin A 1969 J. Phys. B: At. Mol. Phys. 2631

Strand T G and Bonham R A 1964 J. Chem. Phys. 401686 